\section{Paraneoplastic thrombus or relapse of a pulmonary artery sarcoma?}

A 34-year-old man with a previous history of left pneumectomy with curative intent after diagnosis of intimal sarcoma arising from the left pulmonary artery was referred for increasing dyspnoea, and pain at the right shoulder. The patient was free of tumour relapse at nearly 5 years follow-up.

A contrast-enhanced multidetector CT (MDCT) shows a filling defect within the main pulmonary artery (figure 1A). Axial fusion image of integrated $\left[{ }^{18} \mathrm{~F}\right]$ fluorodeoxyglucose positron emission tomography $\left({ }^{18} \mathrm{FDG} \mathrm{PET}\right) / \mathrm{CT}$ confirmed an increased uptake at the same level and additionally at the right scapula, highly suggestive of a metastasis (figure $1 \mathrm{~B}$ ). To relieve the patient's symptoms, and improve his quality of life and his short-term prognosis, a palliative operation was carried out. The main and the right pulmonary arteries were completely resected and successfully reconstructed using a polytetrafluoroethylene (PTFE) vascular prosthesis (Impra Flex, $19 \mathrm{~mm}$ ) with supravalvular anastomosis, and radiotherapy of the osteolytic lesion at the right scapula was performed.

Intraoperatively, a solid whitish-grey intravascular mass with a maximum diameter of $3.0 \mathrm{~cm}$, was identified (figure 1C) and histology confirmed a relapse of a pulmonary artery sarcoma showing abundant malignant spindle cells with high cellularity, frequent mitoses and nuclear pleomorphism (figure 1D).

\section{DISCUSSION}

Pulmonary artery sarcoma is a rare malignancy with a poor prognosis, arising from the mesenchymal cells of the intima of the pulmonary artery.

Invasion of the pulmonary artery by angiosarcoma or other tumour is part of the differential diagnosis of thromboembolic disease. $^{1} 2$

MDCT findings can be specific in patients with advanced disease, particularly in the case of extraluminal tumour extension; other findings favouring the diagnosis of pulmonary artery sarcoma include a low-attenuation filling defect occupying the entire luminal diameter of the proximal or main pulmonary artery and expansion of the involved arteries. ${ }^{3}$ However, these
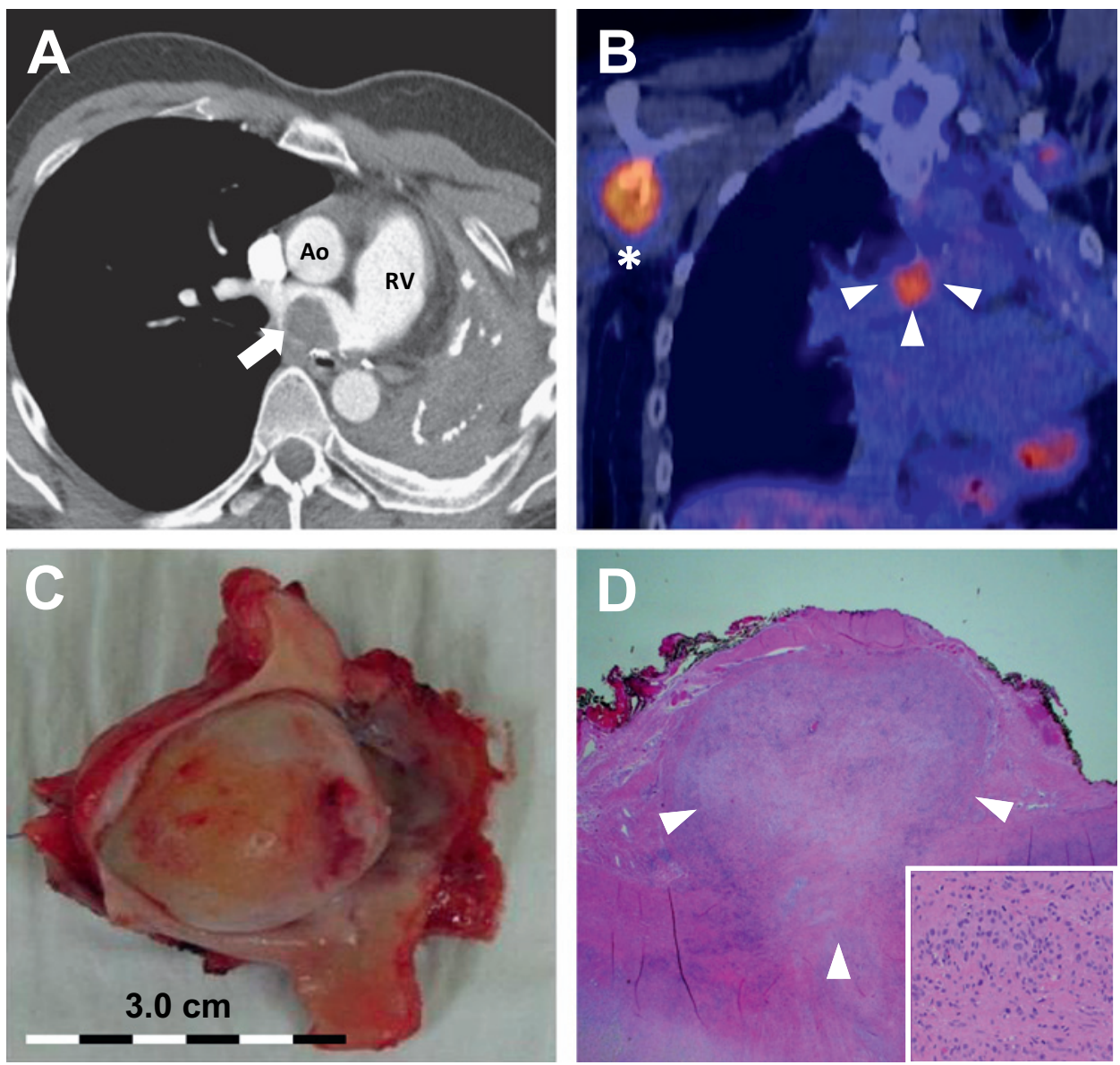

Figure 1 (A) A chest contrast-enhanced multidetector CT image shows an intraluminal filling defect in the main pulmonary artery (arrow) suspicious of a relapse of pulmonary artery sarcoma (maximum diameter of $3.0 \mathrm{~cm})$. The right ventricle is moderately dilated $(4.1 \mathrm{~cm})$. Ao, aorta; RV, right ventricle. (B) Axial fusion image of integrated $\left[{ }^{18} \mathrm{~F}\right]$ fluorodeoxyglucose positron emission tomography $\left({ }^{18} \mathrm{FDG}\right.$ PET)/CT shows increased uptake (standardised uptake value (SUV) of 7.0) in the main pulmonary artery (arrowheads). Note the presence of an osteolytic lesion with high uptake of ${ }^{18} \mathrm{FDG}$ at the level (diameter $3.0 \mathrm{~cm}$ ) of the right shoulder (asterisk) with an SUV measured at 8.4 compatible with a metastasis. (C) Photograph of specimen showing a whitish-grey, intravascular mass (maximal diameter of $3.0 \mathrm{~cm}$ ) within the main pulmonary artery. (D) Photomicrograph of the intravascular mass showing abundant spindle cells with high cellularity, frequent mitoses, nuclear pleomorphism and a high nuclear/cytoplasmic ratio; these findings are suggestive of moderately differentiated sarcoma. The tumour cells were positive for CD31 and vimentin, and negative for pancytokeratin, CD34 and S100. 
MDCT findings can be non-specific in cases of extensive pulmonary thromboembolism. On the other hand, the application of ${ }^{18}$ FDG PET shows negative FDG uptake in the case of blood thrombi, whereas a malignant tumour shows clearly positive FDG uptake. ${ }^{4}$ Therefore, for early and accurate differential diagnosis, ${ }^{5}$ a combined PET/CT scan is found to be useful and should be considered mandatory, since the two conditions need a different therapeutic approach.

\section{LEARNING POINTS}

- The differential diagnosis of obstruction of the pulmonary artery is relatively difficult; invasion of the pulmonary artery by angiosarcoma or other tumour should be considered. $^{12}$

- MDCT findings can be specific for angiosarcoma in patients with advanced disease, particularly in the case of extraluminal tumour extension. ${ }^{3}$

- The finding of a low-attenuation filling defect at MDCT occupying the entire luminal diameter of the proximal or main pulmonary artery can be non-specific in cases of extensive pulmonary thromboembolism.

- A combined CT/PET should be considered mandatory, since the two conditions need a different therapeutic approach. ${ }^{18}$ FDG PET shows negative FDG uptake in the case of blood thrombi, whereas a malignant tumour shows clearly positive FDG uptake. ${ }^{4}$

Michel Oberson, Constanze Sophie Pawelczak, Felix Meincke

University Hospital of Bern, Switzerland

Correspondence to Dr Michel Oberson, University Hospital of Bern, Viale Cassone 32, Lugano-Pregassona 6963, Switzerland; obersonmichel@yahoo.com

Competing interests None.

Patient consent Obtained.

Provenance and peer review Not commissioned; externally peer reviewed. Accepted 15 December 2009

Thorax 2010;65:941-942.

doi:10.1136/thx.2009.124693

\section{REFERENCES}

1. Tschirch FT, Del Grande F, Marincek B, et al. Angiosarcoma of the pulmonary trunk mimicking pulmonary thromboembolic disease. A case report. Acta Radiol 2003:44:504-7.

2. Terra RM, Fernandez A, Bammann $\mathrm{RH}$, et al. Pulmonary artery sarcoma mimicking a pulmonary artery aneurysm. Ann Thorac Surg 2008;86:1354-5.

3. Yi CA, Lee KS, Choe $\mathrm{YH}$, et al. Computed tomography in pulmonary artery sarcoma: distinguishing features from pulmonary embolic disease. Comput Assist Tomogr 2004;28:34-9.

4. Nabi HA, Zubeldia JM. Clinical applications of (18)F-FDG in oncology. J Nucl Med Technol 2002;30:3-9.

5. Kim JH, Gutierrez FR, Lee EY, et al. Primary leiomyosarcoma of the pulmonary artery: a diagnostic dilemma. Clin Imaging 2003;27:206-11. 